\title{
Ray tracing model of the auroral kilometric radiation generation in the 3-D plasma cavity
}

\author{
T. M. Burinskaya \\ Space Research Institute of RAS, Profsoyuznaya str. 84/32, Moscow, 117997, Russian Federation \\ Correspondence to: T. M. Burinskaya (tburinsk@iki.rssi.ru)
}

Received: 3 June 2013 - Revised: 21 August 2013 - Accepted: 28 August 2013 - Published: 7 October 2013

\begin{abstract}
Propagation and amplification of the auroral kilometric radiation (AKR) in a three-dimensional plasma cavity is investigated using the approximation of the geometrical optics, and taking into account both the slightly relativistic electrons propagating inside a cavity and the background cold electrons. It is shown that the global magnetic field inhomogeneity plays a key role in a wave escape from a thin plasma cavity. The main contribution to the AKR spectrum is made by waves initially generated with the component of group velocity directed to the Earth and with the optimum relationship between the wave vector components, controlling the value of the linear grow rate and duration of the ray lifetime inside a source.
\end{abstract}

Keywords. Magnetospheric physics (auroral phenomena)

\section{Introduction}

The auroral kilometric radiation (AKR) is the most powerful nonthermal natural emission from the Earth; its power may be as high as $10^{7}-10^{9} \mathrm{~W}$ (Gurnett, 1974). The AKR has been systematically investigated by experimental and theoretical methods since its discovery by the ELECTRON-2 satellite in 1965. Now it is reliably established that AKR sources are thin cavities oriented tangentially to the auroral oval and aligned with the background magnetic field (Louarn and Le Quéau, 1996a; Mutel et al., 2008). They have a small latitudinal width about several tens of kilometers as compared to their longitudinal extent, which is of the order of 1000 $2000 \mathrm{~km}$. Plasma of AKR sources is tenuous with electron population essentially composed of downward-going slightly relativistic particles with a horseshoe distribution (Ergun et al., 2000). A peculiar feature of the electron distributions observed inside the sources is an accumulation of particles with high transverse energies on the order of several $\mathrm{KeV}$ but low parallel velocities (Louarn and Le Quéau, 1996a). The electron cyclotron maser instability (CMI) (Wu and Lee, 1979) is widely accepted as the fundamental mechanism responsible for generation of the terrestrial AKR, and similar radio emissions from the solar system's planets having their own magnetic field. The AKR is strongly polarized emission generally observed to be generated below the local electron gyrofrequency, and mainly propagating as the extraordinary $X$ mode waves (Hanasz et al., 2000; Panchenko et al., 2008). So in the present investigation we have concentrated on the $X$ mode especially because the electromagnetic energy initially created by the CMI mostly lies on the internal $X$ mode (Louarn and Le Quéau, 1996b). There is a longstanding question arising in studies of cyclotron maser instability in a localized space region with a reduced density: how the radiation generated below the cutoff frequency of the external cold plasma would escape from a source. It is evident that time-dependent processes, as well as plasma and magnetic field inhomogeneities, are of primary importance for understanding the AKR escape from a source region. Taking into account the occurrence of low-frequency waves in the regions of AKR generation (Lund, 2010), the wave exit from a thin plasma cavity with adiabatically slowly varying width has been considered in Burinskaya and Rauch (2012). It was found that due to the increase of the magnetic field and plasma density inside a cavity during the width contraction, the $X$ mode waves have a chance to leave the cavity. The intensity of the outgoing radiation increases during the width contraction and vice versa. A similar correlation between the AKR power and low-frequency oscillations of the magnetic field has been observed by Uozumi et al. (2011). Louarn and Le Quéau (1996b) have qualitatively discussed the escape of AKR from a cavity due to the upward propagation of waves 
inside a source region until their frequency becomes higher than the cutoff frequency of surrounding plasma. The role of magnetic field gradients in wave radiation outwards from a plasma cavity was emphasized by Cairns et al. (2008). However up to now the factors influencing the AKR escape from a source are not clearly understood. Another important problem is associated with theoretical calculations of the wave amplification rates that barely account for the observed spectral intensity of the AKR. In the present study, using the ray tracing approximation and taking into account both background cold electrons and slightly relativistic electrons, we study the wave amplification and propagation in the 3-D finite cavity, and show that the main contribution to the AKR spectrum is made by waves initially generated with the component of group velocity directed to the Earth.

\section{Physical model}

Because of the complexity of the dispersion relation for a horseshoe distribution, a ring distribution of the form $f\left(v_{/ /}, v_{\perp}\right)=\left(2 \pi v_{\perp 0}\right)^{-1} \delta\left(v_{/ /}\right) \delta\left(v_{\perp}-v_{\perp o}\right)$ is used inside a source. Although such a distribution function is idealized, it takes into account relativistic effects and, except a slight increase of the growth rates in comparison with more realistic distributions, the main characteristics of the unstable modes remain almost unchanged (Le Quéau and Louarn, 1989). The propagation of waves is investigated using the equations of the geometrical optics under the condition that the real part of wave frequency is kept constant (Bernstein, 1975).

$$
\begin{aligned}
& \frac{\mathrm{d} \boldsymbol{R}}{\mathrm{d} t}=\frac{\partial D_{\mathrm{R}}}{\partial \boldsymbol{k}} / \frac{\partial D_{\mathrm{R}}}{\partial \omega} \\
& \frac{\mathrm{d} \boldsymbol{k}}{\mathrm{d} t}=-\frac{\partial D_{\mathrm{R}}}{\partial \boldsymbol{R}} / \frac{\partial D_{\mathrm{R}}}{\partial \omega} .
\end{aligned}
$$

Here $\boldsymbol{R}$ is the position vector of a wave packet, $\omega$ is the real part of the wave frequency, $\boldsymbol{k}$ is the wave vector, and $D_{\mathrm{R}}$ is the real part of the dispersion relation $D(\boldsymbol{R}, \omega, \boldsymbol{k})=$ $D_{\mathrm{R}}+i D_{i m}=0$. The growth rate is found as a solution of the dispersion relation at every point along the ray path. Within the geometrical optics approximation, plasma is considered as locally homogeneous, and the dispersion relation may be written as

$$
\left(\frac{\varepsilon_{1}-N_{/ /}^{2}}{\varepsilon_{1}}-N_{\perp}^{2}\right)\left(\frac{\varepsilon_{1}^{2}-\varepsilon_{2}^{2}}{\varepsilon_{1}}-N_{/ /}^{2}-N_{\perp}^{2}\right)=\frac{N_{/ /}^{2} \varepsilon_{2}^{2}}{\varepsilon_{1}^{2}},
$$

where $N_{/ /, \perp}=k_{/ /, \perp} c / \omega(c$ is the speed of light, while subscripts // and $\perp$ symbolize vector components along and perpendicular to the background magnetic field, respectively). Taking into account that in the space region under investigation the electron Langmuir frequency $\omega_{\mathrm{p}}$ is much lower than the electron cyclotron frequency $\omega_{\mathrm{c}}$, and the characteristic wavelength of the excited waves is much larger than the electron gyroradius, the components of the dielectric tensor are found for the ring distribution in Burinskaya and Rauch (2007). Considering both background cold electrons and slightly relativistic electrons in a source region, the permittivity in Eq. (2) has the form $\varepsilon_{1,2}=\varepsilon_{1,2 \text { (cold) }}+\varepsilon_{1,2 \text { (source) }}$, where

$$
\begin{aligned}
& \varepsilon_{1 \text { (cold) }}=1-\frac{\omega_{\mathrm{p}(\text { cold })}^{2}(\boldsymbol{R})}{\omega^{2}-\omega_{\mathrm{c}}^{2}(\boldsymbol{R})} ; \quad \varepsilon_{2(\text { cold })}=\frac{\omega_{\mathrm{p}(\text { cold })}^{2}(\boldsymbol{R}) \cdot \omega_{\mathrm{c}}(\boldsymbol{R})}{\omega\left(\omega^{2}-\omega_{\mathrm{c}}^{2}(\boldsymbol{R})\right)} ; \\
& \varepsilon_{1 \text { (source) }}=1-\frac{\omega_{\mathrm{p}(\text { source })}^{2}(\boldsymbol{R})}{2 \omega^{2}}\left(F_{1}+F_{2}\right) ; \\
& \varepsilon_{2 \text { (source) }}=\frac{\omega_{\mathrm{p}(\text { source })}^{2}(\boldsymbol{R})}{2 \omega^{2}}\left(F_{1}-F_{2}\right) ; \\
& F_{1}=\frac{\omega}{\omega-\omega_{\mathrm{c}}(\boldsymbol{R}) \cdot\left(1-v_{\perp 0}^{2} / 2 c^{2}\right)} \\
& \quad+\frac{v_{\perp 0}^{2}\left(k_{/ /}^{2} c^{2}-\omega \cdot \omega_{\mathrm{c}}(\boldsymbol{R})\right)}{2 c^{2}\left(\omega-\omega_{\mathrm{c}}(\boldsymbol{R}) \cdot\left(1-v_{\perp 0}^{2} / 2 c^{2}\right)\right)^{2}} ; \\
& F_{2}=\frac{\omega}{\omega+\omega_{\mathrm{c}}(\boldsymbol{R}) \cdot\left(1-v_{\perp 0}^{2} / 2 c^{2}\right)} \\
& \quad+\frac{v_{\perp 0}^{2}\left(k_{/ /}^{2} c^{2}-\omega \cdot \omega_{\mathrm{c}}(\boldsymbol{R})\right)}{2 c^{2}\left(\omega+\omega_{\mathrm{c}}(\boldsymbol{R}) \cdot\left(1-v_{\perp 0}^{2} / 2 c^{2}\right)\right)^{2}} .
\end{aligned}
$$

Here $\omega_{\mathrm{p} \text { (cold) }}$ and $\omega_{\mathrm{p} \text { (source) }}$ are plasma frequencies defined by the background plasma density and the density of the source energetic electrons, respectively (see Eqs. 3 and 4 below). The model density-depleted cavity used in the present study has a small latitudinal width with the central magnetic latitude $70^{\circ}$, and is aligned with the magnetic field lines. We perform the ray tracing calculations in the spherical coordinate system $(r, \theta, \varphi)$ with a polar axis coinciding with the magnetic field axis and pointing upwards from the Earth; $r$ is measured from the Earth's center, and $\varphi$ is measured from the plane of symmetry of density-depleted cavity under consideration. The dipolar magnetic field model is adopted in this study. The density profile of the background plasma is taken as

$n_{\text {cold }}(\boldsymbol{R})=\frac{n_{0}}{\left(r / 2 R_{\mathrm{E}}\right)^{2}}\left[1-\exp \left(-\frac{\left(\theta-\theta_{0}\right)^{2}}{\sigma_{\theta}^{2}}-\frac{\left(\varphi-\varphi_{0}\right)^{2}}{\sigma_{\varphi}^{2}}\right)\right]$,

and the density of the source energetic electrons

$n_{\text {source }}(\boldsymbol{R})=\frac{n_{0, \text { source }}}{\left(r / 2 R_{\mathrm{E}}\right)^{2}} \exp \left(-\frac{\left(\theta-\theta_{0}\right)^{2}}{\sigma_{\theta}^{2}}-\frac{\left(\varphi-\varphi_{0}\right)^{2}}{\sigma_{\varphi}^{2}}\right)$.

Here $R_{\mathrm{E}}$ is the Earth radius, $\theta_{0}=20^{0}, \varphi_{0}=0, n_{0}$ is the density of background cold plasma outside the cavity at 
$r=2 R_{\mathrm{E}}, n_{0 \text {,source }}$ is the density of the energetic electrons at $\theta=\theta_{0}, \varphi=\varphi_{0}$ and $r=2 R_{\mathrm{E}}, \sigma_{\theta}$ and $\sigma_{\varphi}$ are the characteristic cavity widths in $\theta$ and $\varphi$ directions. The ions play the role of a neutralizing background. In what follows, we will present the results calculated for the initial parameters adapted to the AKR generation region at $r=2 R_{\mathrm{E}}$, where the local electron cyclotron frequency at the center of the cavity under study is $198 \mathrm{kHz}$. The background density of cold electrons $n_{0}$ is taken as $5 \mathrm{~cm}^{-3}$, the energy and density of source electrons at the center of the cavity and given altitude as $4 \mathrm{KeV}$ and $1 \mathrm{~cm}^{-3}$, respectively, and $\sigma_{\theta}=0.008$ and $\sigma_{\varphi}=0.23$. These parameters fall in the range of experimentally observed values (Louarn and Le Quéau, 1996a).

\section{Simulation results}

The time history of different rays launched at the center of the cavity at $r=2 R_{\mathrm{E}}$ has been studied. The emphasis is placed on waves generated with a reasonable value of the wave vector $\varphi$-component, $k_{\varphi}$, because the cyclotron maser instability driven by a ring or a shell-like velocity distribution has the largest growth rate for the $X$ mode emission near-perpendicular to the magnetic field direction. The main effect of the source finite size is that the radiation is preferentially generated in a direction tangential to the source frontiers (Louarn and Le Quéau, 1996b; Burinskaya and Rauch, 2007). Figure 1 presents the wave packet coordinates versus time normalized to $R_{\mathrm{E}} / c$ for five rays. In what follows, wave vectors are normalized to $\omega_{\mathrm{Hfix}} / c$, and frequencies are normalized to $\omega_{\mathrm{Hfix}}\left(\omega_{\mathrm{Hfix}}\right.$ is the cyclotron frequency at $r=2 R_{\mathrm{E}}, \theta=20^{\circ}$ ). We have explored rays launched with $k_{\theta}=0, k_{\varphi}=0.75$ and different values of $k_{\mathrm{r}}$. The normalized frequencies are $0.9942,0.9945$ and 0.9965 for rays with initial values of $k_{\mathrm{r}}$ equal to $0, \pm 0.25$, and \pm 0.5 , respectively. All these frequencies lie below the cutoff frequency of the background cold plasma calculated at $r=2 R_{\mathrm{E}}$. Recall that $\omega_{\text {cutoff }}=\left(\omega_{\mathrm{H}}+\left(\omega_{\mathrm{H}}^{2}+4 \omega_{\mathrm{pe}}^{2}\right)^{1 / 2}\right) / 2$, where $\omega_{\mathrm{H}}$ and $\omega_{\mathrm{pe}}$ are the local cyclotron and plasma frequencies, respectively. Panel (a) of Fig. 1 shows the time dependence of $r$ coordinate normalized to the Earth's radius for five rays under study. Rays launched with positive values of $k_{\mathrm{r}}$ run upward from the Earth, and those launched with negative $k_{\mathrm{r}}$ initially propagate to the Earth. To gain a better insight into the ray propagation, we add the inset demonstrating ray trajectories in the range $1.995 \leq R<2.015$. The time dependences of $\theta$ and $\varphi$ coordinates are shown in Figs. $1 \mathrm{~b}$ and c, respectively. The ray initially propagating to the Earth reaches a location inside the cavity where its wave radial group velocity becomes zero, and then is reflected upward. During the ray propagation upward from the Earth, the magnitude of the wave vector component parallel to the local magnetic field grows, as shown in Fig. 2. Notice that the geomagnetic field is directed to the Earth in the space region under consideration. The wave
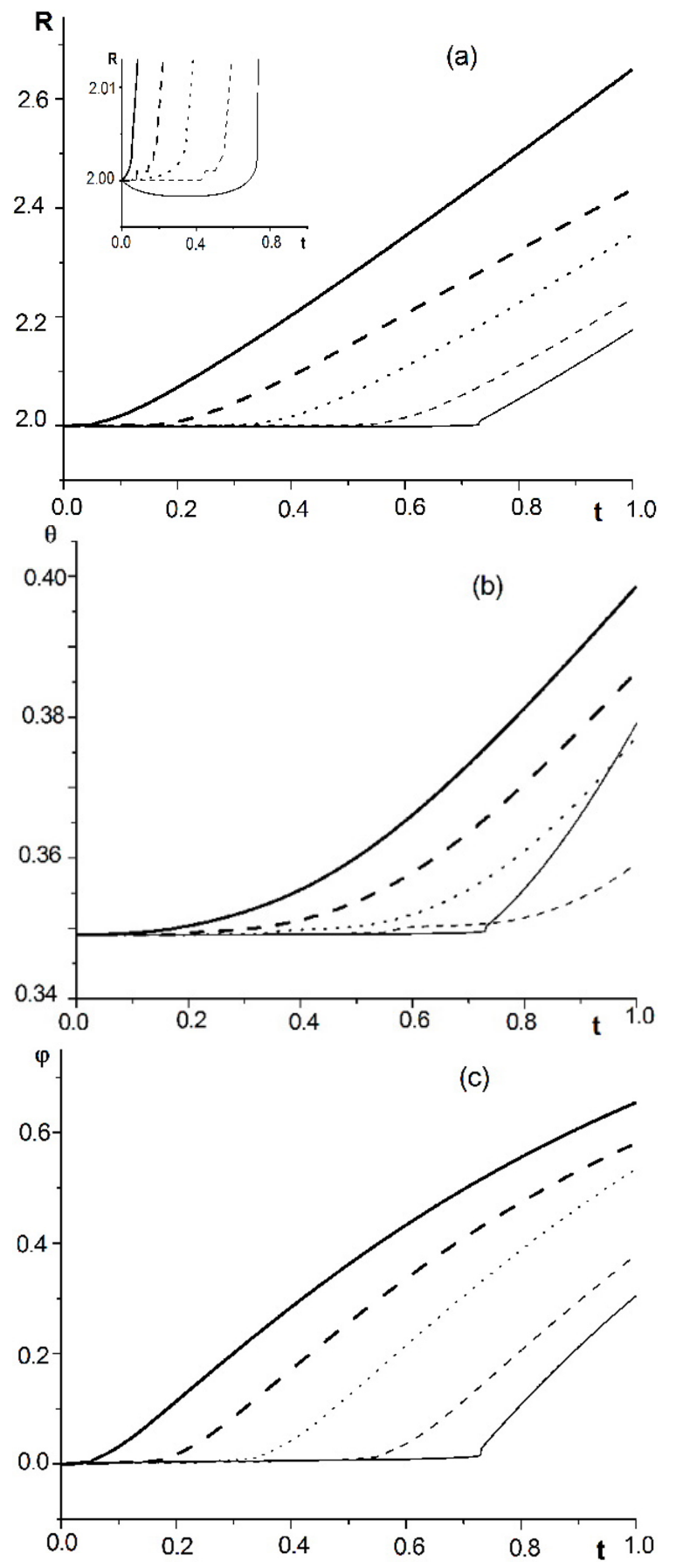

Fig. 1. Spherical coordinates versus time normalized to $R_{\mathrm{E}} / c$ for five wave packets with initial wave vector components $k_{\theta}=$ $0, k_{\varphi}=0.75$ and different values of $k_{\mathrm{r}}$. Thick solid lines correspond to $k_{\mathrm{r}}=0.5$, light solid lines to $k_{r}=-0.5$. Thick dashed lines correspond to $k_{\mathrm{r}}=0.25$, light dashed lines to $k_{\mathrm{r}}=-0.25$, and dotted lines correspond to $k_{\mathrm{r}}=0$. 


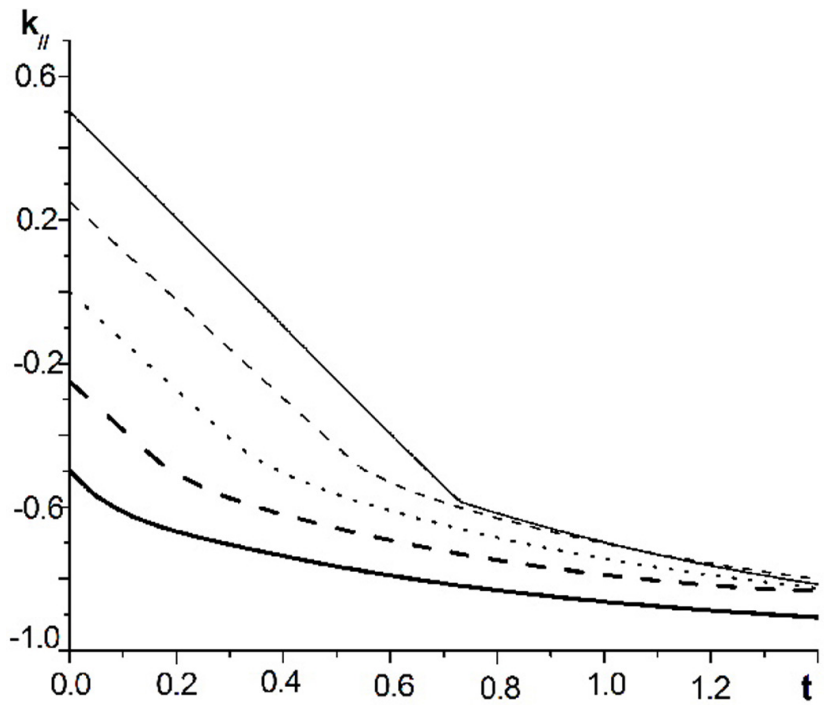

Fig. 2. Wave vector components aligned with the local magnetic field as functions of dimensionless time for the same five rays as in Fig. 1.

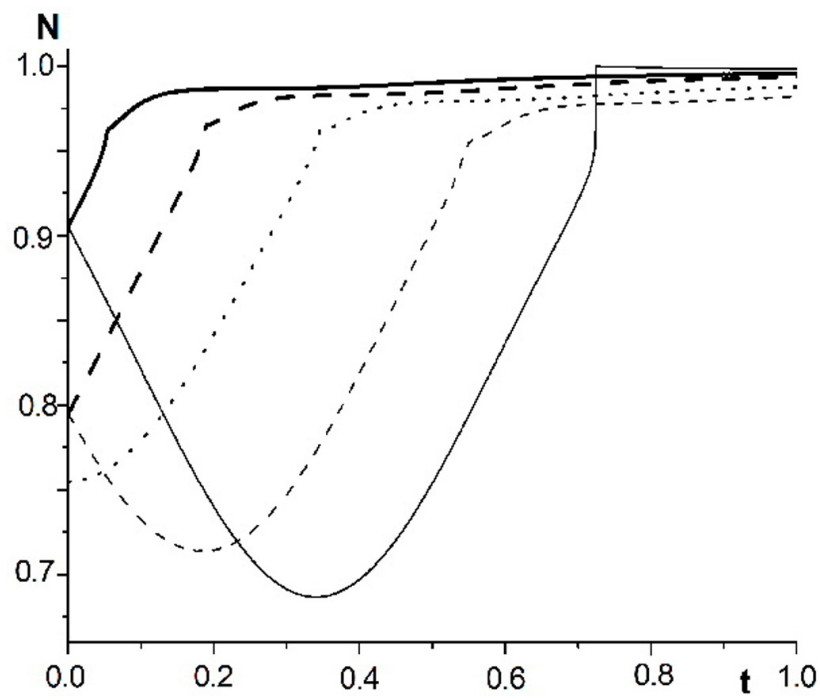

Fig. 3. Wave refractive indexes as functions of dimensionless time for the same five rays as in Fig. 1.

refractive index $N=k c / \omega$ appreciably varies along the ray trajectory, and finally approaches to unity after the ray exit from the source (see Fig. 3). All five rays finally propagate inside the source upward from the Earth until the local cutoff frequency of the surrounding plasma becomes equal to or smaller than the wave frequency. Then the rays leave the cavity and propagate outside the source region. The longer the ray stays inside the source, the more energy it gains. For parameters used in our calculations, multi-reflections of AKR inside a cavity were not observed. A ray double pass inside a source (first downward to the Earth and then upward) is that

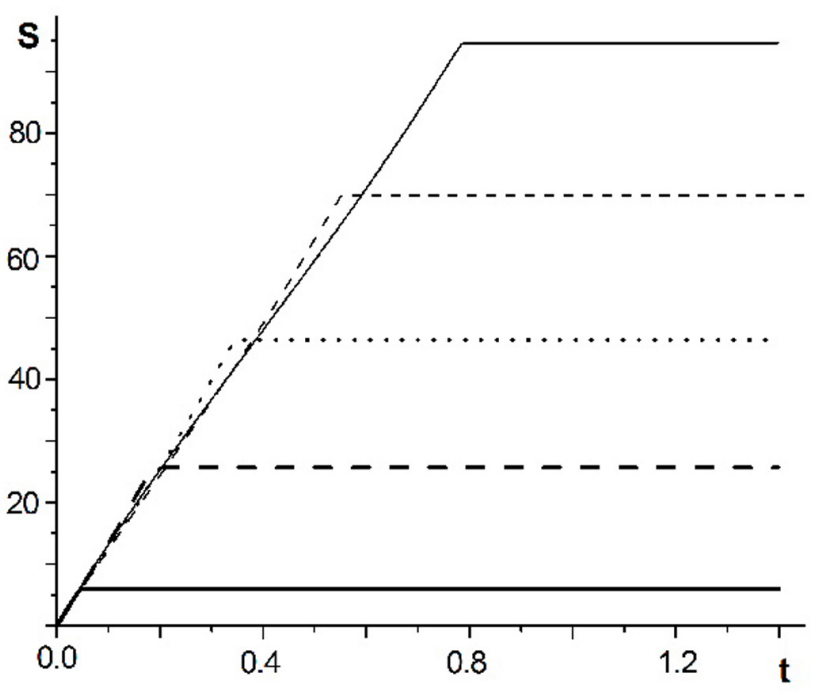

Fig. 4. Amplification factors versus time for the same five rays as in Fig. 1.

which maximizes the ray lifetime inside a source because the radial group velocity is very small in the vicinity of the reflection point. The amplification factor $S=\int_{0}^{t} \gamma(t) \mathrm{d} t$ for five rays under consideration is shown in Fig. 4 as a function of time. It is clearly seen that the energy accumulation may be vastly different for rays with equal frequency. This is the case for the rays with $\omega=0.9965\left(k_{\mathrm{r}}= \pm 0.5\right)$. When the ray initially running to the Earth leaves the source region, its amplification factor is 20 times larger than the amplification factor of the ray propagating upward from its origin. So it is likely that the most pronounced contribution to the AKR spectrum is made by waves initially generated with the component of group velocity directed to the Earth.

The time history of different rays investigated in our simulations has shown a possibility of wave conversion from $X$ to $Z$ mode waves inside a source region. It is well known that in the cold plasma two branches of the dispersion relation corresponding to the extraordinary $X(N<1)$ and $Z$ $(N>1)$ modes are far apart on a dispersion relationship diagram. However there is an additional branch connecting the $X$ and $Z$ modes in a homogeneous relativistic plasma with hot electrons (Cairns et al., 2008). Figure 5 demonstrates the form of the dispersion curves for the perpendicular propagation of extraordinary waves in the homogeneous plasma for a ring distribution with the same parameters as have been used at the midpoint of the model source. Panel (a) shows the relative deviation of real frequency from the electron cyclotron frequency $\delta \omega=\left(\omega-\omega_{\mathrm{H}}\right) / \omega_{\mathrm{H}}$ versus the dimensionless wave number, and panel (b) shows the growth rate normalized to $\omega_{\mathrm{H}}$. A purely real branch with a negative group velocity connecting two unstable branches of the $X$ and $Z$ modes is clearly seen. The case of mode transition in the 

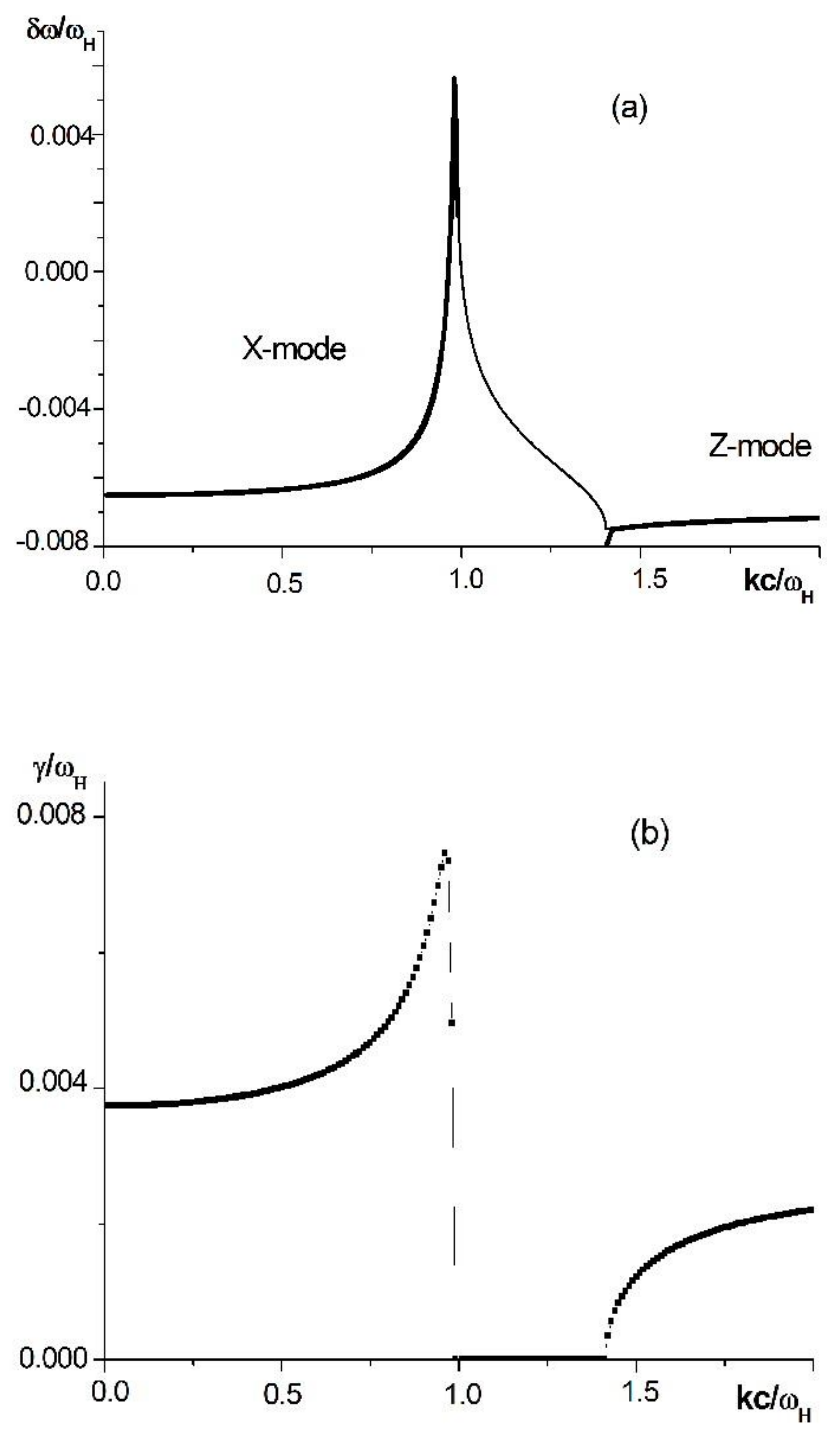

Fig. 5. The dispersion relations for the perpendicular propagation in the homogeneous plasma for a ring distribution with the same parameters as have been taken at the center of the model source. (a) $\delta \omega=\left(\omega-\omega_{\mathrm{H}}\right)$ and (b) growth rates versus the dimensionless wave number.

inhomogeneous plasma is demonstrated in Fig. 6, where the time dynamics of three rays with $k_{\theta}=0, k_{\varphi}=0.95$ and different $k_{\mathrm{r}}$ launched from the cavity center is shown: $k_{\mathrm{r}}=0$ (dotted curves), $k_{\mathrm{r}}=0.2$ (thick solid curves), and $k_{\mathrm{r}}=-0.2$ (light solid curves). The normalized frequencies are 0.9985 and 1.0012 for rays with initial values of $k_{\mathrm{r}}$ equal to 0 and \pm 0.2 , respectively. Note that the normalized cutoff frequency of the background plasma is 1.0099 at the point of ray origin. Panel (a) shows the normalized coordinate $R=r / R_{\mathrm{E}}$ as a function of time. The behavior of rays with the initial value of $k_{\mathrm{r}}=0$ or $k_{\mathrm{r}}=0.2$ is quite similar to the ray propagation discussed above. A completely different type of propagation occurs for the ray with initial component $k_{\mathrm{r}}=-0.2$. Initially
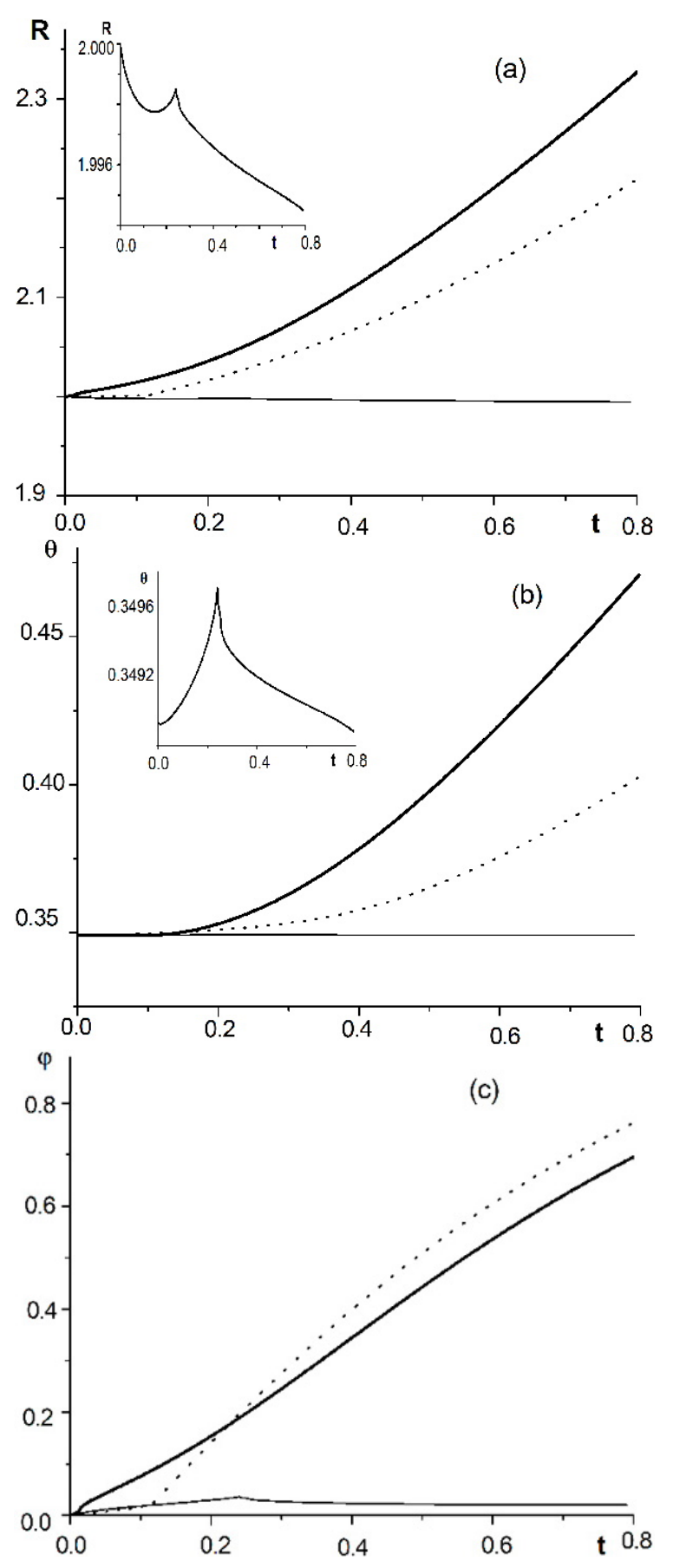

Fig. 6. Spherical coordinates versus dimensional time for three wave packets with initial wave vector components $k_{\theta}=0, k_{\varphi}=$ 0.95 and different values of $k_{\mathrm{r}}$. Thick solid lines correspond to $k_{\mathrm{r}}=0.2$, dotted lines to $k_{\mathrm{r}}=0$, and light solid lines to $k_{\mathrm{r}}=-0.2$. The behavior of the wave packet initially propagating to the Earth $\left(k_{\mathrm{r}}=-0.2\right)$ is additionally shown on the enlarged scale in the insets. 


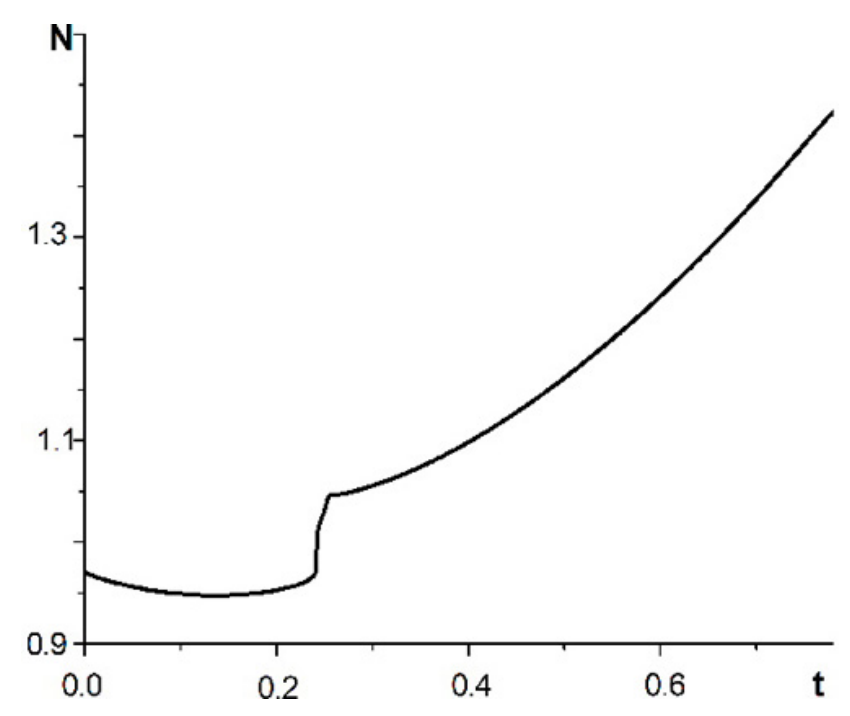

Fig. 7. Wave refractive index versus time for the wave packet initially propagating to the Earth with $k_{\mathrm{r}}=-0.2, k_{\theta}=0$, and $k_{\varphi}=$ 0.95 .

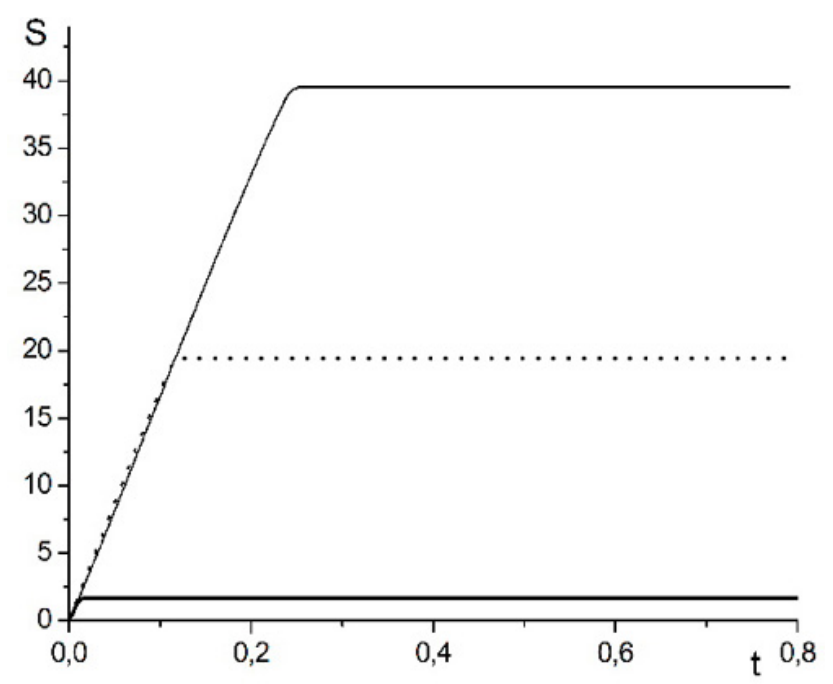

Fig. 8. Amplification factors versus time for the same three rays as in Fig. 6.

this ray, shown by light solid line, moves downward to the Earth and reaches the point of reflection. Then it runs upward but suddenly changes the direction of propagation as shown in the inset in panel (a) and moves downward again. Similar behavior with an angular point of a curve is seen in Fig. 6: inset in panels (b) and (c), where the time dependences of $\theta$ and $\varphi$ coordinates are shown, respectively. The existence of these angular points is representative of sudden change of the group velocity direction, and transition from the $X$ mode dispersion curve to the additional branch connecting the $X$ and $Z$ modes in a homogeneous plasma. The corresponding increase of the wave refractive index is shown in Fig. 7. Rays launched with $k_{\mathrm{r}}=0$ and $k_{\mathrm{r}}=0.2$ propagate from the Earth

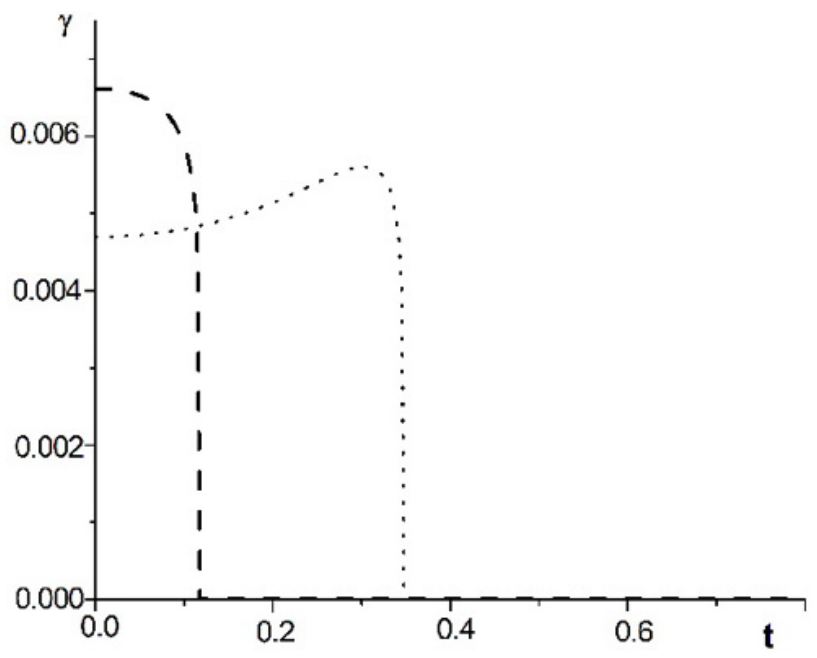

Fig. 9. Growth rates normalized to $\omega_{\mathrm{Hfix}}$ versus dimensionless time for two wave packets launched at the center of the model cavity with $k_{\mathrm{r}}=0, k_{\theta}=0$ and different values of $k_{\varphi}$. Dashed line corresponds to $k_{\varphi}=0.95$, and dotted line corresponds to $k_{\varphi}=0.75$.

inside the source until their frequencies become larger than the local cutoff frequency of the surrounding plasma. Then rays leave the cavity and propagate outside the source region. The ray that has got onto the additional branch of the dispersion relation stays inside the source region during the time under study. The amplification factors for these rays are shown in Fig. 8. The energy storage is stopped when rays leave the source region or when the transition to the additional branch takes place. It is interesting to compare the amplification of rays with initial equal values $k_{\mathrm{r}}=0$ and $k_{\theta}=0$ but different $k_{\varphi}: 0.75$ (Fig. 4) and 0.95 (Fig. 8). Although the linear growth rate for the ray with $k_{\varphi}=0.95(\omega=0.9985)$ is larger than for the ray with $k_{\varphi}=0.75(\omega=0.9942)$, the amplification factor for the latter is more than twice as large as that for the ray with initial component $k_{\varphi}=0.95$. This is because the ray with $\omega=0.9942$ spends more time in the source region (where the wave packet energy is increased, $\gamma>0$ ) than the ray with $\omega=0.9985$ (as shown in Fig. 9).

\section{Discussion}

We have considered the propagation and amplification of fast extraordinary $X$ mode waves inside a thin 3-D cavity taking into account both the source energetic and the background cold electron populations. The distribution function of slightly relativistic electrons in a source region is taken as a ring distribution because the use of more realistic distribution leads to a much more complicated dispersion relation. However, although such a distribution is idealized, the use of the ring distribution in the calculations of dispersion relation in homogeneous plasma gives very similar results to those obtained with a horseshoe distribution as shown in Cairns et 
al. (2008). The propagation of waves is investigated within the approximation of geometrical optics, with the components of the dielectric tensor calculated in the locally homogeneous plasma approximation. The dipolar magnetic field model is adopted in our study.

The time history of different rays launched at the center of the model cavity in Eq. (4) at $r=2 R_{\mathrm{E}}$ has been studied. It is shown that there is a possibility of wave transition from the $X$ mode extraordinary wave to the $Z$ mode wave via the additional branch of the dispersion relation arising in the hot relativistic plasma. However, this transition occurs in a rather short time interval, and the wave refractive index is changed from $N<1$ to $N>1$ within a distance of the order of several wave lengths. Therefore, to gain a better insight into this conversion process and calculate the transmission coefficient in an inhomogeneous plasma and determine the modification of the polarization parameters of the AKR emissions when the wave transition occurs, the complete set of Maxwell equations must be used.

The investigation of wave amplification inside the source region has shown that while the cyclotron maser instability has the largest growth rate for the $X$ mode wave generation in the immediate vicinity of $\varphi$-direction, the role of $k_{\mathrm{r}}$ component in the accumulation of wave energy is very important too. The ray launched with a group velocity directed to the Earth would initially propagate downward until it reaches a reflection point where its group velocity becomes zero. After reflection the ray propagates upward inside the source until the cutoff frequency of the surrounding background plasma decreasing with altitude due to the global magnetic field inhomogeneity becomes equal to, or smaller than, the ray frequency. The longer a ray propagates inside the source region, the more energy it gains. It has previously been discussed that the wave amplification factor $\sim 20$ may be considered as a sufficient one to reproduce the observed levels of the AKR emissions (Omidi and Gurnett, 1984). Calculations of the wave amplifications along the trajectory made in several papers have shown that there is a problem achieving this value (Omidi and Gurnett, 1984; Zarka et al., 1986; Gaelzer et al., 1994). But in these studies the possibility for wave packets to make double passage inside a source region due to the wave reflection has not been considered. The amplification factors obtained in our calculations may be well in excess of this value (see Fig. 4). However, we have used a ring distribution function that leads, as we have mentioned above, to the increase of grow rates. For precise comparison with experimental data, it is necessary to use the more realistic horseshoe distribution. Nevertheless, the conclusion of our studies is that the main contribution to the AKR spectra is given by waves initially generated with a component of the group velocity directed to the Earth, and with the optimum relationship between the $k_{\varphi}$ and $k_{\mathrm{r}}$ controlling the value of the linear grow rate and a ray lifetime inside a source. It should be noted that in our calculations all rays are refracted upward during their propagation from the Earth inside the source region, as well as when they leave it. These model results are strongly supported by experimental observations demonstrating that the AKR-radiating diagram is anisotropic in the plane perpendicular to the geomagnetic field. It is highly confined latitudinally, typically $\pm 20^{\circ}$ from the magnetic field direction, and is much wider in the $\varphi$-direction along the cavity (Mutel et al., 2008; Mogilevsky et al., 2008). The answer to the question of whether the AKR diagram is a hollow cone or not requires calculations with a wide range of initial ray parameters, and is currently under the study.

Acknowledgements. The study was supported by the Ministry of Education and Science of the Russian Federation, projects 8413 and 8527, as well as a grant of RFBR 12-02-92614 KO.

Topical Editor C. Jacobi thanks two anonymous referees for their help in evaluating this paper.

\section{References}

Bernstein, I. B.: Geometric optics in space- and time-varying plasmas, Phys. Fluids, 18, 320-324, 1975.

Burinskaya T. M. and Rauch, J. L.: Waveguide regime of cyclotron maser instability in plasma regions of depressed density, Plasma Phys. Rep., 33, 28-37, doi:10.1134/S1063780X07010047, 2007.

Burinskaya, T. M. and Rauch, J.-L.: Auroral kilometric radiation from a nonstationary thin plasma cavity, Ann. Geophys., 30, 1093-1097, doi:10.5194/angeo-30-1093-2012, 2012.

Cairns, R. A., Vorgul, I., and Bingha, R.: Cyclotron maser radiation from an inhomogeneous plasma, Phys. Rev. Lett., 101, 215003, doi:10.1103/PhysRevLett.101.215003, 2008.

Ergun, R. E., Carlson, C. W., McFadden, J. P., Delory, G. T., Strangeway, R. J., and Pritchett, P. L.: Electron-cyclotron maser driven by charged-particle acceleration from magnetic fieldaligned electric fields, Astrophys. J., 538, 456-466, 2000.

Gaelzer, R., Ziebell, L. F., and Schneider, R. S.: Ray tracing studies on auroral kilometric radiation in finite width auroral cavities, J. Geophys. Res., 99, 8905-8916, 1994.

Gurnett, D. A.: The Earth as a radio source: Terrestrial kilometric radiation, J. Geophys. Res., 79, 4227-4238, 1974.

Hanasz, J., Boudjada, M. Y., Schreiber, R., Krawczyk, Z., Malycha, M., Mogilevsky, M. M., Rucker, H. O., and Romantsova, T. V.: Dynamic spectra of the Stokes parameters for the dayside and nightside Auroral Kilometric Radiation, Geophys. Res. Lett., 27, 1631-1634, 2000.

Le Quéau, D. and Louarn, P.: Analytical study of the relativistic dispersion: application to the generation of the auroral kilometric radiation, J. Geophys. Res., 94, 2605-2616, 1989.

Louarn, P. and Le Quéau, D.: Generation of the auroral kilometric radiation in plasma cavities-I. Experimental study, Planet. Space Sci., 44, 199-210, 1996a.

Louarn, P. and Le Quéau, D.: Generation of the auroral kilometric radiation in plasma cavities-II. The cyclotron maser instability in small size sources, Planet. Space Sci., 44, 211-224, $1996 \mathrm{~b}$.

Lund, E. J.: On the dissipation scale of broadband ELF waves in the auroral region, J. Geophys. Res., 115, A01201, doi:10.1029/2009JA014545, 2010. 
Mogilevsky, M. M., Romantsova, T. V., Hanasz, J., Burinskaya, T. M., and Schreiber, R.: On the source of auroral kilometric radiation, JETP Lett., 86, 709-711, 2008.

Mutel, R. L., Christopher, I. W., and Pickett, J. S.: Cluster multispacecraft determination of AKR angular beaming, Geophys. Res. Lett., 35, L07104, doi:10.1029/2008GL033377, 2008.

Omidi, N. and Gurnett, D. A.: Path-integrated growth of auroral kilometric radiation, J. Geophys. Res., 89, 10801-10812, 1984.

Panchenko, M., Hanasz, J., and Rucker, H. O.: Estimation of linear wave polarization of the auroral kilometric radiation, Radio Sci., 43, RS1006, doi:10.1029/2006RS003606, 2008.
Vozumi, T., Yumoto, K., Tokunaga, T., Solovyev, S. I., Shevtsov, B. M., Marshall, R., Liou, K., Ohtani, S., Abe, S., Ikeda, A., Kitamura, K., Yoshikawa, A., Kawano, H., and Itonaga, M.: AKR modulation and global Pi2 oscilation, J. Geophys. Res., 116, A06214, doi:10.1029/2010JA016042, 2011.

Wu, C. S. and Lee, L. C.: Theory of the terrestrial kilometric radiation, ApJ., 230, 621-626, 1979.

Zarka, P., Le Quéau, D., and Genova, F.: The maser synchrotron instability in an inhomogeneous medium: Determination of the spectral instability of auroral kilometric radiation, J. Geophys. Res., 91, 13542-13558, 1986. 